Revue internationale P.M.E.

Économie et gestion de la petite et moyenne entreprise

\title{
L'exportation des PME québécoises et l'accord de libre-échange avec les États-Unis
}

\section{André Joyal, Pierre-André Julien et Laurent Deshaies}

Volume 6, numéro 1, 1993

URI : https://id.erudit.org/iderudit/1008164ar

DOI : https://doi.org/10.7202/1008164ar

Aller au sommaire du numéro

Éditeur(s)

Presses de l'Université du Québec

ISSN

0776-5436 (imprimé)

1918-9699 (numérique)

Découvrir la revue

Citer cet article

Joyal, A., Julien, P.-A. \& Deshaies, L. (1993). L'exportation des PME québécoises et l'accord de libre-échange avec les États-Unis. Revue internationale P.M.E., 6(1), 67-85. https://doi.org/10.7202/1008164ar
Résumé de l'article

En relation avec les espoirs véhiculés et les difficultés signalées dans la littérature sur les PME exportatrices, l'article présente une partie des résultats d'une recherche effectuée en vue de mesurer la sensibilité à l'Accord de libreéchange des PME manufacturières de trois régions du Québec. Pour les PME dont les activités sont touchées par l'Accord, nous montrons dans quelle mesure elles profitent de cette ouverture du marché soit pour s'engager sur la voie de l'exportation ou pour accroître leur engagement sur le marché américain. Un profil des firmes exportatrices, ou intéressées à le devenir, est également présenté à partir de différentes variables telles la taille, les investissements, la veille technologique et commerciale et le recours aux ressources du milieu.
Tous droits réservés @ Presses de l’Université du Québec, 1993
Ce document est protégé par la loi sur le droit d'auteur. L’utilisation des services d'Érudit (y compris la reproduction) est assujettie à sa politique d'utilisation que vous pouvez consulter en ligne.

https://apropos.erudit.org/fr/usagers/politique-dutilisation/ 


\title{
L'exportation des PME québécoises et l'accord de libre-échange avec les États-Unis
}

\author{
André JOYAL \\ Pierre-André JULIEN \\ Laurent DESHAIES* \\ Université du Québec à Trois-Rivières
}

\begin{abstract}
RÉSUMÉ
En relation avec les espoirs véhiculés et les difficultés signalées dans la littérature sur les PME exportatrices, l'article présente une partie des résultats d'une recherche effectuée en vue de mesurer la sensibilité à l'Accord de libreéchange des PME manufacturières de trois régions du Québec. Pour les PME dont les activités sont touchées par l'Accord, nous montrons dans quelle mesure elles profitent de cette ouverture du marché soit pour s'engager sur la voie de l'exportation ou pour accroître leur engagement sur le marché américain. Un profil des firmes exportatrices, ou intéressées à le devenir, est également présenté à partir de différentes variables telles la taille, les investissements, la veille technologique et commerciale et le recours aux ressources du milieu.
\end{abstract}

\begin{abstract}
Given the hoper and difficulties found in the literature on exporting by small businesses, this article presents the results of a study undertaken to measure the impact of the Free Trade Agreement on the manufacturing sector in three regions of Quebec. For the small enterprises that are affected by the Agreement, we show that they use this opportunity to begin exporting or to increase their sales on the American market. A profile of these firms is also given in terms of variables such as size, investment, environmental scanning and use of external resources.
\end{abstract}

* Les auteurs sont membres du Groupe de recherche en économie et gestion des PME (GREPME) de l'Université du Québec à Trois-Rivières. Ils remercient Richard Lachance et Martin Morin qui furent leurs assistants de recherche. Adresse : GREPME, Département d'administration et d'économique, C.P. 500, Trois-Rivières (Québec), G9A 5H7. 


\section{RESUMEN}

En relación con las expectativas transmitidas y las dificultades señaladas en la literatura sobre las PME exportadoras, el artículo presenta una parte de los resultados de una investigación efectuada con la finalidad de medir la sensibilidad al Tratado de Libre Comercio de las PME manufactureras de tres regiones de Quebec. Para las PME, cuyas actividades son afectadas por el Tratado, presentamos en que medida ellas se benefician de esta apertura del mercado, ya sea para comprometerse por medio de la exportación o para aumentar su compromiso con el mercado americano. Se presenta igualmente un perfil de las firmas exportadoras o interesadas en el porvenir, a partir de las diferentes variables, tales como el tamaño, las inversiones, la tecnología vieja y comercial, y echar mano a los recursos del medio.

\section{Introduction}

L'accord de libre-échange (ALE) intervenu entre le Canada et les États-Unis, et en vigueur depuis le $1^{\text {er }}$ janvier 1989, exercera une influence sur l'évolution des petites et moyennes entreprises appartenant à plusieurs branches industrielles. En bonne partie favorables à l'ouverture des frontières, les milieux d'affaires québécois y voient avant tout une source de nouveaux débouchés leur permettant de bénéficier de certaines économies d'échelle dont l'exiguïté du marché national les prive généralement. Étant donné le peu de temps écoulé depuis l'entrée en vigueur de cet accord, à ce jour, peu d'études ont été faites sur ses conséquences pour les PME. Il existe bien une une somme de documentation servant à mieux comprendre l'ALE et son impact sur l'économie canadienne et québécoise. Il s'agit, avant tout, d'études macro-économiques issues de travaux du Conseil économique du Canada, du gouvernement du Québec de 1'Institut C. D. Howe (Schwanen, 1992) ou de différentes associations sectorielles. Or, il importe d'évaluer l'impact de l'ALE sur les PME manufacturières et ses effets sur l'attitude et le comportement de leurs dirigeants.

Mis à part des études de plusieurs chercheurs se rapportant aux PME face à l'exportation, des contributions comme celles de Dana (1990) et de Ragman et Verbeke (1989) comptent parmi les rares analyses dignes de mention. Alors que le premier voit dans cette nouvelle stratégie industrielle une occasion qui offre de grandes possibilités pour les PME dans la mesure où les entrepreneurs seront bien préparés, les seconds estiment que la clé du succès réside dans l'exploitation de certains créneaux bien spécifiques.

En relation avec les espoirs véhiculés et les mises en garde exprimés dans la littérature sur les PME exportatrices, cet article présente des résultats d'une recherche sur la mesure du degré de sensibilité à l'ALE des PME manufacturières de trois régions du Québec. 
Même si elle semble une aventure remplie de promesses, l'exportation demeure toujours une voie exigeante et jonchée d'obstacles. Avant même d'être à la portée des entreprises dynamiques désireuses d'élargir leur marché, elle comporte des difficultés de plusieurs ordres. Aux facteurs conjoncturels habituels s'ajoutent des problèmes propres aux marchés extérieurs et des obstacles inhérents à la nature de la PME. De plus, les conditions nécessaires au maintien de la compétitivité sur les marchés internationaux requièrent de la part des entreprises des efforts soutenus au plan de la recherche et du développement, de la veille technologique et commerciale, de l'innovation et exigent enfin, des ressources humaines et financières importantes. Dans ces conditions, les exportateurs, nouveaux et expérimentés, se doivent de recourir à l'aide externe, soit par l'entremise des différents services gouvernementaux ou par celle de réseaux partenariaux dans leur environnement immédiat ou hors des frontières.

Les travaux portant sur les difficultés à exporter rencontrées par les PME mettent en évidence une gamme d'éléments, dont plusieurs comme on le verra, s'appliquent aux entreprises considérées dans le cadre de la présente recherche.

Pour expliquer la faiblesse des exportations dans le chiffre d'affaires des PME, différents facteurs sont relevés tels l'absence d'information sur les marchés étrangers (Miesenbrock, 1988 ; Phillippe, 1990 ; Ali et Swiercz, 1991 ; Howard et Herremans, 1988), l'insuffisance des ressources financières ; la faible familiarité avec les programmes d'aide gouvernementale, la complexité des mesures se rapportant aux tarifs étrangers, la fluctuation des monnaies, les coûts de transport et de la mise en marché, les contingentements d'importation, le poids des réglementations différentes, auxquels facteurs s'ajoutent bien sûr des obstacles d'ordre culturel et linguistique pouvant affecter les communications avec les clients ou le choix d'un distributeur (Kathawala et al., 1989; Naumann et Lincoln, 1991 ; Kaynak et Ghauri, 1987). Trop peu de services pour surmonter ces obstacles sont mis à la disposition des petites entreprises et ceux qui existent sont coûteux, compliqués et restrictifs.

Sur les marchés locaux et plus encore sur les marchés internationaux, le maintien de la compétitivité comporte une foule d'exigences. En effet, des efforts constants s'imposent au plan de la $\mathrm{R}$ et $\mathrm{D}$, de la veille technologique et commerciale, de l'innovation et de la gestion des ressources humaines (Julien et Marchesnay, 1988 ; Julien, 1993). Il est vrai qu'au Canada, $20 \%$ des dépenses en $R$ et D seraient effectuées par des petites entreprises (Bulloch, 1991) et celles qui investissent dans les nouvelles technologies sont mieux placées pour affronter la concurrence. En effet, Lefebvre et Lefebvre (1988) ont montré que les entreprises favorables à l'ALE utilisaient plus d'applications simultanées en technologie de l'information et de production que celles qui s'y opposent. Par exemple, dans notre enquête, $25 \%$ des entreprises favorables à l'ALE recourent à la $\mathrm{CAO}$ contre seulement $12 \%$ pour les entreprises moins favorables. 
Pour contrer les obstacles et satisfaire les exigences requises pour le maintien de la compétitivité de leur entreprise, les exportateurs peuvent recourir à de l'aide technique dispensée par les différents organismes responsables de la mise en application des programmes gouvernementaux. Ainsi, $85 \%$ (selon un échantillon non aléatoire) des firmes étudiées par Chenier et Prince (1990) ont utilisé à un moment ou un autre une forme d'aide gouvernementale pour leurs exportations. Par contre, d'autres études soulignent la faible familiarité des entrepreneurs envers ces divers programmes (Kathawala et al., 1989). Une telle situation s'explique en partie par la façon dont cette aide est distribuée. Erwin (1989) montre que, contrairement aux attentes, les firmes ne séparent pas leurs besoins d'assistance à l'exportation en catégories fonctionnelles comme le marketing ou la finance, mais perçoivent plutôt ce besoin considérant les particularités techniques associées à l'exportation.

Cette assistance peut, entre autres choses, toucher les canaux de distribution. Ceux-ci dépendent d'une grande quantité de facteurs : la fréquence des ventes, leur régularité, leur importance, la valeur unitaire du produit, le nombre et la répartition des consommateurs, le nombre et la différence des produits exportés, la similitude des marchés et la distance les séparant, et enfin, la disponibilité des ressources financières et managériales. On comprend que la demande des entreprises manufacturières pour l'aide à l'exportation tend à se centrer, après l'assistance au financement, sur deux éléments majeurs, soit l'élargissement des marchés et le choix de représentants et/ou de distributeurs, comme le montrent Chenier et Prince (1990). Ces derniers font ressortir qu'au Québec et en Ontario, les deux types de services les plus utilisés se rapportent aux visites commerciales à l'aide des programmes gouvernementaux mis de l'avant par les gouvernements de ces deux provinces.

Dans les pages qui suivent, nous expliquons d'abord la démarche utilisée et donnons les principales caractéristiques de l'échantillon ayant servi de support. Ensuite, à la lumière des informations recueillies, nous montrons dans quelle mesure les PME, dont les activités sont concernées par l'ALE, profitent de cette nouvelle ouverture du marché américain, soit en envisageant la possibilité d'exporter ou, s'il y a lieu, en augmentant la part des produits expédiés outre frontière. Nous présentons subséquemment un profil des firmes exportatrices et de celles intéressées à le devenir à partir de différentes variables telles la taille, les investissements, la veille technologique et commerciale, le recours aux ressources du milieu, etc. ; autant d'éléments qui permettent de relier la performance des entreprises aux aptitudes à exporter. Enfin, nous situons les entreprises exportatrices de l'échantillon suivant leur perceptions vis-à-vis leurs concurrentes américaines. 


\section{Méthodologie}

Si l'on admet que les entrepreneurs devraient connaître l'ALE afin d'être en mesure de réagir à ses conséquences par une attitude défensive d'une part, ou pour en tirer un avantage par une réaction offensive d'autre part, il est intéressant de constater que $54 \%$ des entreprises exportatrices enquêtées ont adopté différentes mesures spécifiques pour mieux faire face à la nouvelle situation suscitée par l'ALE contre seulement 13,6\% de la part des entreprises non exportatrices. Quant aux entreprises intéressées par le marché de l'exportation, elles sont $40 \%$ à avoir réagi d'une façon ou d'une autre, ce qui montre bien l'existence d'une sensibilité particulière au changement ou d'un certain dynamisme de la part des entreprises exportatrices ou désireuses de le devenir. Cependant, pour ces entreprises, à l'instar de leurs homologues de par le monde, la voie est parsemée d'embûches.

Pour y voir plus clair, quelques éléments d'informations méthodologiques faciliteront la compréhension de résultats présentés par notre étude.

L'ensemble des PME étudiées dans cette enquête appartiennent à trois régions fort disparates. Deux d'entre elles se situent près de Montréal et participent dans une certaine mesure à l'évolution de l'économie de la métropole québécoise. La troisième, située au « cœur du Québec » se caractérise par un secteur manufacturier particulièrement concentré ; cette dernière comprend en fait deux sous-régions différentes séparées par le fleuve Saint-Laurent. Sa partie sud, marquée par une plus grande ruralité, a néanmoins développé un dynamisme qui permet l'association avec d'autres régions reconnues par la vivacité de leurs PME.

Trois grands critères ont guidé le choix des branches industrielles retenues :

1. L'importance des secteurs dans l'une et l'autre des trois régions, en termes d'emplois et de nombre d'entreprises.

2. La place occupée par les PME à l'intérieur d'un secteur donné : déterminée par le nombre d'entreprises en excluant les très petites (moins de 5 employés) et le pourcentage des emplois dans les entreprises de 5 à 199 employés par rapport à l'emploi total généré par l'ensemble des entreprises du secteur.

3. L'importance des produits concernés par l'ALE : déterminée par le nombre de produits identifiés par l'accord, la catégorie d'échelonnement de l'abolition de la barrière tarifaire (entre 5 et 10 ans) et l'étendue de la protection canadienne imposée avant l'accord. 
Les industries retenues sont les suivantes: trois industries de l'alimentation (code101-viande et volailles, 104-produits laitiers, 107-boulangerie); tout le vêtement (24), presque la totalité de l'industrie du bois (25), le meuble (26), deux industries du papier (27), l'industrie de l'impression commerciale (281), la machinerie (31) et enfin, celui des embarcations (328).

Une pré-enquête directe fut effectuée à l'hiver 1991 auprès d'un nombre limité (30) d'entreprises réparties dans chacune des régions et produisant des biens concernés par l'ALE. Ce test a servi d'assise à l'enquête postale réalisée au début de l'été 1991 auprès de l'ensemble des entreprises visées, soit un total de 976 entreprises. Le questionnaire, divisé en quatre parties, comprenait 51 questions fermées et 11 questions ouvertes. Une première partie visait à obtenir des renseignements généraux alors qu'une deuxième se rapportait à l'état de la productivité et à la situation financière. Dans les deux autres parties, on cherchait à percevoir les attitudes envers l'ALE et les caractéristiques de l'environnement des entreprises.

Ainsi, 242 (taux de réponse de 27,5\%) entreprises ont complété et retourné le questionnaire.

\section{Profil général des entreprises de l'échantillon}

Le tableau 1 décrit l'échantillon par rapport à la population. On voit, en ce qui concerne la taille des entreprises, que le plus haut taux $(31,6 \%)$ de réponse provient des entreprises ayant entre 5 et 19 employés, soient 158 entreprises sur un total possible de 509. Les autres catégories de taille s'échelonnent comme suit : 24,3\% pour les 20 à 49 employés, 20,8\% pour les 50 à 99 employés et $20,6 \%$ pour les entreprises ayant entre 100 et 250 employés. Comme $30 \%$ des entreprises ont vu leurs effectifs baisser de 1987 à 1990, il s'ensuit qu'un certain nombre de répondants ayant déclaré de 5 à 19 employés faisaient partie auparavant d'une classe supérieure de population, soit celles des 20-49 employés, ce qui aurait pour effet de gonfler les classes inférieures au détriment des classes supérieures. Il en est de même pour les entreprises de la catégorie suivante.

Les tests de représentativité effectués simultanément pour les quatre catégories de taille et les 21 industries ne peuvent conclure à un quelconque biais de l'échantillon vis-à-vis-la population. Ainsi, l'échantillon d'entreprises sur lequel s'appuient nos résultats peut être jugé suffisamment représentatif de la population en général, et ce, par rapport à la provenance régionale, à la taille et aux secteurs d'activité. 


\section{TABLEAU 1}

Population totale, nombre de répondants et $\%$ de réponses

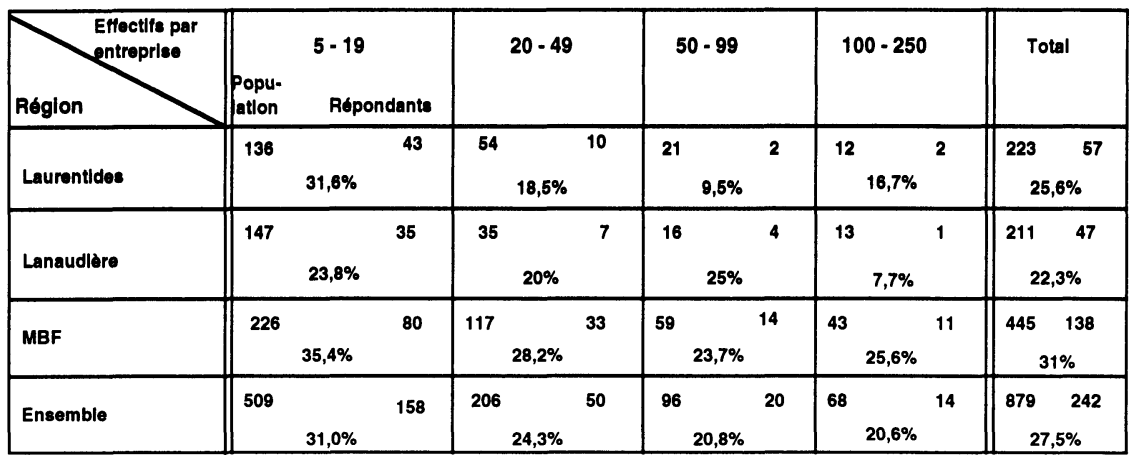

Plus de $55 \%$ des répondants ont moins de 50 employés. Le nombre moyen d'employés par entreprise est de 28 , dont $22,5 \%$ affectés à la production. Le salaire horaire moyen des employés est de 10,15\$. Comme on le verra plus loin, en relation avec l'évaluation du degré de dynamisme des entreprises, seulement un peu plus du quart des répondants (27\%) affirment s'impliquer dans la $\mathrm{R}$ et $\mathrm{D}$, et moins des deux tiers de ceux-ci le font de façon systématique. La grande majorité des entreprises avancent différentes raisons pour justifier leur absence d'implication dans ce domaine: insuffisance de besoins (20\%) ou de ressources financières et humaines $(25 \%)$.

Pour ce qui est de la veille commerciale, cette dimension dont l'importance devient de plus en plus évidente et, bien sûr, reliée au dynamisme des entreprises, elle ne préoccupe que $35 \%$ des entreprises. Les efforts dans ce domaine ne se font que de façon sporadique en vue d'acquérir de l'information sur de nouveaux produits et de nouveaux marchés. Quant à la veille technologique, elle touche $41 \%$ des répondants dont les deux cinquièmes ne s'y consacrent qu'au besoin.

Près de la moitié des répondants considèrent que l'information reçue sur l'ALE est incomplète ou insuffisante. Les différents médias écrits ou électroniques constituent la principale source d'information; viennent ensuite le gouvernement fédéral, les revues spécialisées, les associations sectorielles et le gouvernement du Québec. Dans l'ensemble, il appert que les entreprises ne se sentent pas très concernées par l'ALE et ne soient pas non plus très informées sur le sujet. Constatations d'autant plus étonnantes que le nombre d'entreprises touchées par l'ALE, pour au moins un produit, s'élève à 164 , soit $70 \%$ de l'échantillon alors que la totalité de la production de $33 \%$ des entreprises est touchée et que $57 \%$ des entreprises ont des produits concernés par l'accord pour plus de la moitié de leur production. 


\section{Profil particulier des entreprises exportatrices et désireuses d'exporter}

Dans notre échantillon $18,2 \%$ soit 44 entreprises exportent à l'extérieur du Canada (alors que la moyenne québécoise en 1989 est 14,4 \% (BSQ, 1992). Vingt-neuf pourcent des répondants déclarent être intéressés à exporter. Ainsi, 52,3\% des PME enquêtées n'exportent pas et n'envisagent pas de le faire.

Les raisons évoquées pour ne pas exporter varient beaucoup, allant de la trop petite taille, à la sous-capitalisation, aux difficultés de transport, et enfin à toutes les raisons évoquées plus haut par différents auteurs. En fait, il semble que la peur de s'engager dans l'exportation caractérise bon nombre d'entrepreneurs. Le temps et l'information font cruellement défaut aux yeux de plusieurs. En outre, trop peu d'entrepreneurs prennent conscience des avantages que recèlent les marchés étrangers. Face au marché américain, ils craignent de ne pas pouvoir répondre aux commandes effectuées et ne réagissent parfois qu'en présence d'une situation de survie.

Quant aux entreprises exportatrices de notre échantillon, elles sont engagées dans de telles opérations depuis 13 ans en moyenne. La part moyenne de leur chiffre d'affaires représentée par les exportations est de $21 \%$. Ayant divisé nos entreprises en trois grandes catégories suivant qu'elles se consacrent à l'assemblage, à la transformation ou à la fabrication ${ }^{1}$, on constate que les premières exportent à $20 \%$ alors que les deux autres présentent des proportions respectives de $26 \%$ et $15 \%$.

Amesse et Zaccour (1989), dans leur étude sur les PME québécoises ont montré que la taille de la firme constitue un facteur significatif de différenciation entre exportateurs et non exportateurs, le pourcentage des entreprises exportatrices ayant tendance à croître régulièrement avec la taille. Alors qu'une firme sur quatre ayant moins de 15 employés est exportatrice, cette proportion atteint les $50 \%$ pour celles ayant entre 25 et 50 employés tandis qu'elle atteint les deux tiers pour les entreprises de 50 employés et plus. Ils révèlent, de plus, que le profil des firmes exportatrices se différencie fortement de celui des firmes non exportatrices.

Nos données nuancent ce constat trop général en signalant l'existence d'un seuil. En effet, la taille moyenne de nos entreprises exportatrices est de 54,6.

1. Classification CTI : Assemblage : $\mathrm{n}^{\text {os }} 281,311,312,319,328$; transformation : $\mathrm{n}^{\text {os }} 273,279,101,104,107,251,252,259$; fabrication : $\mathrm{n}^{\text {os }} 243,244,245,249$, 254, 261, 264, 269. 
Parmi nos entreprises ayant entre 50 et 250 employés, la proportion des exportatrices atteint les $51,5 \%$ alors qu'elle n'atteint que les $26 \%$ parmi celles dont les effectifs varient entre 20 et 49 employés et seulement $12,8 \%$ pour les entreprises dont le nombre d'employés varie entre 10 et 19. Le tableau 2 présente le nombre d'entreprises exportatrices par rapport à l'étendue de leur effectif.

TABLEAU 2

Exportation et nombre d'employés

\begin{tabular}{lrrrrrr}
\hline $\begin{array}{l}\text { Nombre } \\
\text { d'employés }\end{array}$ & $\begin{array}{c}\text { Entreprises } \\
\text { non exportatrices }\end{array}$ & \multicolumn{2}{c}{$\begin{array}{c}\text { Entreprises } \\
\text { intéressées } \\
\text { à exporter }\end{array}$} & \multicolumn{2}{c}{$\begin{array}{c}\text { Entreprises } \\
\text { exportatrices }\end{array}$} \\
\hline $5-9$ & 53 & $67,9 \%$ & 21 & $26,9 \%$ & 4 & $0,5 \%$ \\
$10-19$ & 38 & $48,7 \%$ & 30 & $38,4 \%$ & 10 & $12,8 \%$ \\
$20-49$ & 23 & $38,3 \%$ & 14 & $23,3 \%$ & 13 & $21,6 \%$ \\
$50-99$ & 7 & $36,8 \%$ & 2 & $10,5 \%$ & 10 & $52,6 \%$ \\
$100-250$ & 4 & $36,3 \%$ & 3 & $27,2 \%$ & 7 & $63,6 \%$ \\
\hline TOTAL & $\mathbf{1 2 5}$ & & $\mathbf{7 0}$ & & $\mathbf{4 4}$ & \\
\hline
\end{tabular}

L'intérêt envers l'exportation, comme on le voit, croît avec la taille des entreprises. C'est à partir de 50 employés que le nombre d'entreprises exportatrices cesse d'augmenter sensiblement. Il semble donc que ce soient les entreprises ayant entre 10 et 20 employés qui manifestent un véritable intérêt envers l'exportation même si un nombre relativement faible, à l'intérieur de cette catégorie, s'engagent dans l'exportation. Parmi les entreprises de la catégorie suivante (de 20 à 50 employés), les entrepreneurs passent aux actes en concrétisant leurs intentions d'exporter. Au delà de 50 employés, les entreprises paraissent fixées ; elles ne s'interrogent plus sur la place que peuvent occuper les exportations; au contraire, elles cherchent à consolider leurs acquis. À l'opposé, les entreprises ayant moins de 10 employés n'envisagent pas d'élargir leur marché au delà des frontières. Le tableau 3 présente un profil partiel de ces trois catégories d'entreprises.

Alors que moins de $20 \%$ des entreprises non exportatrices pratique la veille commerciale de façon systématique, cette proportion atteint les $41 \%$ pour les entreprises exportatrices. Quant à la $\mathrm{R}$ et $\mathrm{D}$, la différence est également très significative puisque le tiers des entreprises intéressées à exporter y ont recours de même que $53,7 \%$ des exportatrices contre seulement $13,8 \%$ pour les entreprises non exportatrices. 
TABLEAu 3

Comparaison des entreprises suivant leur statut eu égard à l'exportation

\begin{tabular}{llll}
\hline & $\begin{array}{l}\text { Non } \\
\text { exportatrices }\end{array}$ & $\begin{array}{l}\text { Intéressées } \\
\text { à exporter }\end{array}$ & Exportatrices \\
\hline $\begin{array}{l}\text { Participation } \\
\text { à un réseau }\end{array}$ & $20 / 120: 16,7 \%$ & $21 / 70: 30 \%$ & $18 / 42: 42$ \\
$\begin{array}{l}\text { Veille commerciale } \\
\text { de façon systématique }\end{array}$ & $21 / 106: 19,8 \%$ & $14 / 64: 21,8 \%$ & $18 / 41: 43,9 \%$ \\
$\begin{array}{l}\text { Entreprises qui font } \\
\text { de la R et D }\end{array}$ & $16 / 116: 13,8 \%$ & $22 / 64: 34,4 \%$ & $22 / 41: 53,7 \%$ \\
$\begin{array}{l}\text { Investissements passés } \\
\text { et futurs (prévus) } \\
\text { par employé }\end{array}$ & $13501 \$$ & $23340 \$$ & $17874 \$$ \\
$\begin{array}{l}\text { Formation universitaire } \\
\text { du dirigeant }\end{array}$ & $19 / 125: 15,2 \%$ & $25 / 70: 35,7 \%$ & $23 / 44: 52,3 \%$ \\
\hline
\end{tabular}

En ce qui regarde l'implication à l'intérieur de différents réseaux d'entraide, nos résultats confirment une observation de Burton et Schlegelmilch (1987) voulant que les exportateurs ont des contacts soutenus avec d'autres entreprises ou divers organismes. Ainsi, $40,9 \%$ de nos exportateurs contre seulement $16,7 \%$ pour les non-exportateurs affirment entretenir des relations régulières avec différents intervenants. Alors que le tiers des exportateurs ont des contacts réguliers hors de leur région, cette proportion tombe à $2,9 \%$ avec les entreprises non exportatrices. Ces mêmes auteurs avaient mis en évidence le niveau supérieur de formation des cadres supérieurs ou des dirigeants d'entreprise: avec $52 \%$ d'entre eux qui ont reçu une formation universitaire, les dirigeants d'entreprises exportatrices surclassent les non-exportatrices, dont seulement $15,2 \%$ sont dirigées par des entrepreneurs dotés d'une telle formation.

Beamish et Munro (1985) ont dégagé une forte corrélation entre l'importance de l'exportation et la performance des PME en Ontario. Une relation qui semble se vérifier avec les entreprises de notre échatillon. En effet, $75 \%$ de l'ensemble des entreprises ont connu une croissance de leur chiffre d'affaires sur une période de trois ans, et $85 \%$ des entreprises exportatrices se classent parmi celles-ci. Quant aux entreprises intéressées à exporter, en prenant la variable «profit par employé », elles se positionnent mieux que les entreprises qui n'exportent pas (11 691 \$ par rapport à 10421 \$). Pas moins de $69 \%$ de ces mêmes entreprises intéressées par l'exportation ont connu une croissance de leur chiffre d'affaires. Faut-il en déduire que le succès économique suscite un 
intérêt pour l'exportation? En fait, compte tenu du trop grand nombre de variables susceptibles d'expliquer le succès, force est de reconnaître que la majorité des études ne parviennent pas à établir une relation étroite entre la performance des entreprises et leur propension à exporter.

Néanmoins, à partir d'une analyse discriminante effectuée à partir de deux grandes variables, le degré de sensibilité à l'ALE et le dynamisme des entreprises, il a été possible de les diviser en trois grandes catégories et de voir ainsi comment se situent les entreprises exportatrices. La première variable se rapporte à l'importance de la production touchée par l'ALE alors que la seconde synthétise cinq sous-variables (dont certaines sont présentées ci-dessous) couvrant autant de caractéristiques susceptibles de révéler le degré de dynamisme de l'entreprise, les voici :

- les mesures particulières prises par l'entreprise pour contrer ou profiter de l'ALE ;

- la nature de son intégration dans le milieu environnant ;

- les comportements stratégiques ;

- la degré d'attention portée aux aspects commerciaux et technologiques ;

- l'importance relative des investissements effectués ou prévus.

S'il existe une corrélation positive entre ces cinq variables, elle n'est pas assez forte pour que leur utilisation simultanée soit redondante : chaque variable ajoute de l'information nouvelle que l'on ne pouvait trouver chez les autres. Le choix de ces variables a été inspiré, à l'aide d'une analyse factorielle d'une part et par nos connaissance empiriques des PME, d'autre part.

En considérant chaque entreprise selon son degré de sensibilité et son dynamisme il a été possible de classer nos entreprises en trois catégories:

1) les entreprises peu ou pas menacées par l'ALE ;

2) les entreprises vulnérables ;

3) les entreprises très vulnérables ${ }^{2}$.

Le tableau 4 présente les trois catégories d'entreprises suivant leur vulnérabilité : non vulnérables, vulnérables et très vulnérables.

2. Les résultats complets de l'analyse de la vulnérabilité font l'objet d'une publication à part. Ils seront présentés dans l'article rédigé par P.A. Julien, A. Joyal et L. Deshaies (1993). 
Les entreprises exportatrices à $45 \%$ se retrouvent dans le groupe 2 , c'est-à-dire celui des entreprises passablement vulnérables, alors qu'elles sont virtuellement absentes du groupe de firmes très menacées $(4,5 \%)$. Les firmes exportatrices vulnérables se retrouvent dans quatre secteurs menacés (vêtement, meuble, papier, embarcations). Quant aux firmes intéressées par l'exportation, leur répartition entre les groupes 1, 2, et 3 est respectivement 50,0\%,21,4\% et $28,6 \%$ Alors que ces dernières ont une même proportion d'entreprises dans le groupe $1(50,0 \%)$, celles-ci diffèrent sensiblement à l'intérieur des deux groupes.

La différence entre les entreprises exportatrices et non exportatrices jugées non vulnérables n'est pas très forte. Le fait que l'ALE ne représente pas une menace pour plusieurs entreprises non-exportatrices s'explique surtout par la solidité de leur implantation dans leur marché environnant. À moyen terme, leurs arrières sur le marché local semblent assurés.

TABleau 4

Répartition des entreprises suivant leur degré de vulnérabilité

\begin{tabular}{lcccccc}
\hline Vulnérabilité & \multicolumn{2}{c}{ Non vulnérables } & \multicolumn{2}{c}{ Vulnérables } & \multicolumn{2}{c}{ Très vulnérables } \\
\hline Non exportatrices & 73 & $(58,4 \%)$ & 21 & $(16,8 \%)$ & 31 & $(24,8 \%)$ \\
Intéressées à exporter & 35 & $(50,0 \%)$ & 15 & $(21,4 \%)$ & 20 & $(28,6 \%)$ \\
Exportatrices & 22 & $(50,0 \%)$ & 20 & $(45,5 \%)$ & 2 & $(4,5 \%)$ \\
\cline { 2 - 7 } & $\mathbf{1 3 0}$ & $\mathbf{( 5 4 , 0} \%)$ & $\mathbf{5 6}$ & $\mathbf{( 2 3 , 4} \%)$ & $\mathbf{5 3}$ & $\mathbf{( 2 2 , 2} \%)$ \\
\hline
\end{tabular}

En ce qui a trait à l'opinion des entreprises concernant l'ALE, il est intéressant de noter que $63 \%$ des répondants ont affirmé être en faveur de l'ALE lors du débat qui a précédé son adoption alors que seulement $28 \%$ ont soutenu une position contraire ; $9 \%$ des exportateurs ont exprimé leur indifférence. Par ailleurs, entre 1987 et 1990, on constate un léger déplacement de l'opinion, car $6 \%$ des opinions sont passées de favorables à défavorables. Pour la même période, le nombre des indifférents est resté stable. L'entrée du Mexique dans l'ALE suscite cependant plus de craintes chez les entreprises, soit pour $44 \%$ d'entre elles. Parmi les 16 entreprises exportatrices qui ont signalé que l'ALE aurait des effets bénéfiques pour leur entreprise, plus de la moitié (10) ont mentionné l'avantage d'accéder à de nouveaux marchés. Deux entreprises ont signalé une baisse possible du prix des matières premières et deux ont affirmé que la nouvelle situation les obligerait à devenir plus compétitives. Enfin, deux entreprises ont reconnu un avantage relié à la diminution des droits de douane. 
Parmi les 22 entreprises (soit la moitié des entreprises exportatrices) qui ont signalé que l'ALE aurait des effets négatifs, 13 d'entre elles conviennent que l'augmentation de la concurrence et la pression des entreprises américaines leur fera perdre de clients ; 7 autres affirment être déjà défavorisées par la force du taux de change du dollar canadien.

Parmi les facteurs favorables et défavorables à l'évolution de leur entreprise, l'ALE se classe au sixième rang des facteurs négatifs et au troisième des facteurs positifs. La croissance du secteur et les taux d'intérêt (au moment de l'enquête) sont dans l'ordre, les deux facteurs que les répondants ont jugé avoir le plus d'effets positifs sur leur entreprise. Parmi les facteurs négatifs, la récession se classe en premier suivi de la valeur du dollar canadien. En résumé, la récession et le taux de change sont de toute évidence plus déterminants pour l'activité économique des entreprises que l'ALE.

\section{Comportement et stratégies des entreprises exportatrices face à I'ALE}

Seulement $39 \%$ des entreprises exportatrices ont déclaré avoir adopté des mesures pour tirer profit de l'ALE alors que $31 \%$ ont préféré prendre des mesures pour se protéger des éventuelles conséquences négatives.

Les entreprises qui recourent aux ressources du milieu ont pris des mesures dans une proportion plus forte $(70 \%)$ que celles qui s'en abstiennent $(42 \%)$. Les ressources dont il est ici question se rapportent à des institutions ou à des instances gouvernementales responsables des programmes d'aide ou à des composantes d'un réseau de personnes ou de regroupements sectoriels. La plupart des mesures adoptées peuvent être considérées comme des mesures dites «pro-actives » dans le sens qu'elles sont plus offensives que défensives, faisant appel à un dynamisme renouvelé de l'entreprise plutôt qu'à un repli sur elle-même.

À la faveur d'une question ouverte, 24 entreprises exportatrices ont précisé la nature de leur réaction ce qui nous a permis de répertorier un total de 34 mesures que nous avons réparties en quatre catégories.

1. Les mesures relatives au produit (huit mesures : $23,5 \%$ )

On se réfère à des initiatives orientées vers une amélioration de la qualité, une plus grande spécialisation du produit, un changement en faveur du haut de gamme, un effort de design et d'innovation, la mise en marché d'un nouveau produit ou le développement de nouveaux produits adaptés au marché américain. 
2. Les mesures relatives à la productivité (neuf mesures : $23,5 \%$ )

Il s'agit de mesures adoptées en vue d'augmenter la productivité. Elles concernent la modernisation des équipements, la rationalisation de la maind'œuvre (diminution et formation) et la diminution des coûts généraux de production.

3. Les mesures relatives au marché (quatroze mesures : 41,2\%)

On vise par ces initiatives à percer le marché américain à partir d'une exploration des possibilités à la faveur de contacts avec des organismes d'interface (délégation du Québec, foires industrielles) ou par la recherche d'un représentant outre-frontière, le recours à des distributeurs américains, la mise en opération d'un réseau de distribution ou l'envoi de vendeurs aux États-Unis. Les « missions commerciales » apparaissent comme un outil convoité par un nombre grandissant d'entreprises.

4. Les mesures relatives à la structure (trois mesures : 8,8\%)

Ce type de mesures se rapporte aux efforts stratégiques, à plus long terme, déployés par certaines entreprises qui, par exemple, envisagent la possibilité de s'établir aux États-Unis, de se fusionner avec un exportateur, de s'associer avec une entreprise américaine, ou encore d'acheter un compétiteur ou une entreprise américaine.

Le tableau 5 montre que le fait d'exporter ou d'y être intéressé semble prédisposer à l'adoption de mesures visant à tirer profit de l'ALE. Du moins, des taux de $31,0 \%$ et $27,9 \%$, suivant que l'on est exportateur ou intéressé à le devenir, tranchent beaucoup par rapport au $8,7 \%$ des entreprises non exportatrices qui ont adopté des mesures en vue d'en profiter également ou tout simplement pour se prémunir contre ses conséquences.

TABLEau 5

Mesures pour profiter de l'ALE selon le statut face à l'exportation

\begin{tabular}{llcc}
\hline Exportation & Non & Oui & n \\
\hline Pas exportateurs & $91,3 \%$ & $8,7 \%$ & 126 \\
Intéressé par l'exportation & $72,1 \%$ & $27,9 \%$ & 68 \\
Exportateurs & $69 \%$ & $31 \%$ & 42 \\
\hline TOTAUX & $\mathbf{8 1 , 8} \%$ & $\mathbf{1 8 , 2} \%$ & $\mathbf{2 3 6}$ \\
\hline
\end{tabular}

Source: Rapport OPDQ. 
Suivant les différentes catégories de mesures, le tableau 6 montre comment celles-ci se répartissent, toujours en fonction de la situation des entreprises eu égard à l'exportation.

\section{TABLEAU 6}

Types de mesures prises selon le statut face à l'exportation

\begin{tabular}{lccccc}
\hline Statut à l'exportation & \multicolumn{4}{c}{ Nombre de mesures dynamiques prises } \\
& Produit & Production & Marché & Structure & TOTAL \\
\hline N'exporte pas & $16,6 \%$ & $57,7 \%$ & $22,2 \%$ & $5,5 \%$ & $100 \%$ \\
& $(3)$ & $(10)$ & $(4)$ & $(1)$ & $(18)$ \\
Intéressé & $13 \%$ & $38,7 \%$ & $42 \%$ & $6,3 \%$ & $100 \%$ \\
par l'exportation & $(4)$ & $(12)$ & $(13)$ & $(2)$ & $(31)$ \\
Exportateur & $21,3 \%$ & $27,3 \%$ & $42,4 \%$ & $9 \%$ & $100 \%$ \\
& $(7)$ & $(9)$ & $(14)$ & $(3)$ & $(33)$ \\
\hline
\end{tabular}

Source: Rapport OPDQ.

On voit que les entreprises qui n'exportent pas concentrent surtout leurs efforts sur des mesures relatives à la production $(57,7 \%)$. Pour leur part, les entreprises exportatrices mettent l'accent sur des mesures se rapportant au marché $(42,4 \%)$ alors que celles intéressées à exporter partagent leurs efforts dans ces deux catégories de mesures $(38,7 \%$ et $42,0 \%)$. Il est à noter que les entreprises exportatrices, même si elles consacrent beaucoup d'énergie sur des mesures concernant le marché, semblent répartir leurs efforts dans les autres fonctions de l'entreprise, soit $21,3 \%$ pour le produit et $27,3 \%$ pour la production.

\section{Avantages et désavantages par rapport aux concurrents américains}

Les entreprises ont été invitées à préciser les deux principaux avantages et les deux principaux désavantages pour elles par rapport à leurs concurrents américains. Comme pour la section précédente, il s'agissait de deux questions ouvertes auxquelles le répondant pouvait répondre. Les informations recueillies se rapportaient aux éléments suivants :

a) Le produit

La qualité du produit représente l'avantage majeur sur les produits américains. En effet, l'avantage concurrentiel s'exprime surtout à travers le produit 
dont la qualité facilite l'exportation; des efforts sont consentis en ce sens. L'exclusivité et l'innovation constituent les autres avantages reliés au produit. Par ailleurs, le prix de vente des produits américains s'avère un réel désavantage pour les entreprises étudiées.

b) La productivité

À ce chapitre, les répondants estiment faire face à des coûts généraux, de main-d'œuvre et de matières premières plus élevés que leurs concurrents américains, lesquels sont par ailleurs avantagés par les volumes de production.

c) Le marché

Les entreprises se partagent ici en deux groupes égaux : celles qui se considèrent désavantagées par le marché et celles qui peuvent en tirer parti. La qualité du service semble être l'avantage concurrentiel le plus marqué.

d) La conjoncture

C'est sous cette rubrique que les entreprises étudiées présentent le plus de désavantages vis-à-vis leurs homologues américaines. Il s'agit de facteurs qui relèvent essentiellement de la macro-économie. Ainsi, la force du dollar canadien et l'importance des taux d'intérêt, (au moment de l'enquête) des taxes et des exigences fiscales sont mentionnées le plus souvent.

\section{Conclusion}

En ayant classé les entreprises de l'échantillon suivant leur degré de vulnérabilité à partir de leur sensibilité à l'ALE (nombre de produits affectés) et leur dynamisme (suivant cinq sous-variables), on constate que seulement $4,5 \%$ des entreprises exportatrices sont vraiment menacées. Tout comme pour pour les entreprises intéressées à exporter, $50 \%$ des entreprises exportatrices ne sont nullement menacées par l'ALE. Cette situation s'explique, comme nous l'avons vu, par le fait que ce sont ces entreprises qui ont pris des initiatives afin de tirer profit des effets bénéfiques de l'ALE. En faisant preuve d'un dynamisme certain, ces entreprises envisagent l'avenir avec optimisme.

Nous avons également montré l'existence d'un seuil relativement à l'effectif au delà duquel les entreprises s'engagent davantage dans les activités exportatrices. S'il est normal de s'attendre à une relation étroite entre le volume des exportations et la dimension des entreprises, nos données révèlent un intérêt très marqué lorsque celles-ci ont entre 20 et 50 employés. Le nombre d'entreprises exportatrices ne s'accroît pas de manière sensible une fois atteint le seuil 
des 50 employés. En outre, ces dernières paraissent plus à l'aise face au défi que représente la conquête de nouveaux marchés, ce qui ne veut pas dire qu'elles soient indifférentes au support offert par les différents programmes gouvernementaux.

L'insuffisance d'appuis à l'exportation est apparue comme un très sérieux problème pour les entrepreneurs interrogés par Opena (1983). Or, notre enquête révèle que seulement 9 des 63 programmes relevés concernent la mise en marché. Les entreprises qui ont le potentiel et la volonté d'exporter connaissent l'ensemble des programmes accessibles mais trop souvent ces derniers pèchent par leur manque de souplesse. Plusieurs répondants, parmi les entreprises exportatrices, ont exprimé le souhait d'une meilleure adaptation des programmes à leurs besoins. Ainsi, dans l'attribution des programmes, on devrait prendre en considération la démarche globale de l'entreprise de même que le fait qu'elle est unique et se trouve à un stade d'évolution qui lui est particulier.

Cette adaptation des programmes gouvernementaux sera facilitée par une meilleure connaissance des stades de croissance des PME exportatrices que Bilker et Tesar (1977) ont, en leur temps, mis en évidence et qui allait d'un désintéressement total à un engagement sur une base continue. Cependant, leur modèle se prête mal à toute forme d'opérationnalisation et ne permet pas de déterminer la proportion des entreprises qui persistent de manière à devenir de véritables exportatrices. En fait, si l'on sait que les motivations à exporter déterminent dans une certaine mesure le type de stratégies mises de l'avant par les entreprises, plusieurs zones grises demeurent.

Il importe donc, à partir des informations recueillies dans le cadre de cette étude, de s'interroger sur trois types particuliers d'exportation:

- celle due à un concours de circonstances, appelée «par à coup »;

- celle dont la valeur demeure toujours marginale dans l'ensemble du chiffre d'affaires ;

- celle dont la place dans l'ensemble des ventes est capitale.

Pour chacun de ces types, il s'agit d'établir un lien avec l'origine de l'acte d'exportation, avec les décisions stratégiques qui s'y rapportent, avec les conséquences sur l'organisation interne (fonctions de l'entreprise) et externe (alliances stratégiques, réseau de distribution, etc.). Enfin, en confrontant chaque type d'exportation avec les ressources internes (information, financement, études de marché) et les ressources externes (pouvoirs publics, réseaux d'information, maisons de commerce, etc.), il deviendra possible de formuler des recommandations susceptibles de permettre aux autorités gouvernementales une meilleure adaptation de leurs programmes de soutien. 


\section{Bibliographie}

ALI, A. et P.M. SWIERCZ (1991), «Firm size and export behavior : lessons from the Midwest », Journal of Small Business Management, vol. 29, n² 2, avril 1991, p. 71-78.

AMESSE, F. et G. ZACCOUR (1989), « Les gestionnaires et l'exportation : différences de perception et de caractéristiques entre gestionnaires de firmes exportatrices et non exportatrices », Cahiers du CETAI, n 89-18, décembre.

B.S.Q. (1992), Statistiques des PME manufacturières au Québec, Édition 1992, Les Publications du Québec.

BEAMISH, P.W. et J.H. MUNRO (1985), « Export characteristics of small canadian manufacturers », Journal of Small Business and Entrepreneurship, vol. 3, $n^{\circ} 1$, été, p. 93-98.

BILKEY, W.J. et G. TESAR (1977), « The export behavior of smaller-sized Wisconsin manufacturing firms ", Journal of International Business Studies, vol. 8, p. 93-98.

Bulloch, J. F., « Competiting in the global economy : a small business perspective », Journal of Small Business Management, p. 3-8.

Burton, F.N. et SCHLEGElmilch (1987), « Profile analyses of non-exporters versus exporters grouped by export involvement », Management International Review, vol. 27 , p. $38-48$.

CHENIER, J.A. et J.P. PRINCE (1990), « Aid for small business exporting firms : the role of governments and information networks », The Institute of Research on Public Policy, 148 p.

DANA, L.P. (1990), « The Canada-United States Free Trade Agreement and Its Implications for Small Business », Journal of Small Business Management, vol. 28, $\mathrm{n}^{\circ} 2$, avril, p. 64-69.

Erwin, M. E. (1989), Export Assistance Needs of Non-Exporting Small and MediumSized U.S. Firms, Dissertation abstracts international, volume 51/02-A, 132 pages.

HowARD, D.G. et I.M. HERREMANS (1988), « Sources of assistance for small business exporters : advice from succesfull firms », Journal of Small Business Management, vol. $26, \mathrm{n}^{\circ} 3$, juillet, p. 48-54.

JULIEN, P.A. (1993), Information technologique et compétitivité des PME, Paris, OCDE (à paraître).

Julien, P.A., A, Joyal et L. Deshaies (1993), « SMEs and international competition : free trade agreement or globalization? », Journal of Small Business Management (à paraître). 
Julien, P.A. et M. MARchesnay (éd.) (1988), La petite entreprise, Paris ; VuibertGestion.

Kathawala, Y. et all., (1989), "Exporting practices and problems of Illinois firms », Journal of Small Business Management, vol. 27, $\mathrm{n}^{\circ}$ 1. p. 53-59.

Kaynak, E., P.N. Ghauri et E. OlofsSON-Bredenlov (1987), « Export behavior of small swedish firms », Journal of Small Business Management, vol. 25, $\mathrm{n}^{\circ} 2$, avril, p. 26-32.

LeFEBVRE, L.A. et E. LeFEBVRE (1988), « Technologie et libre-échange, une complicité souhaitable ", L'actualité économique, vol. 64, n 4, décembre, p. 616-629.

MIESEMBOCK, K.J., (1988) «Small businesses and exporting : a literature review », International Small Bunisess Journal, Ja/Ma, p. 42-61.

NAUMANN, E. et D.J. LiNCOLN (1991), « Non-tariff barriers and entry strategy alternatives », JSBMS, vol. 29, $\mathrm{n}^{\circ}$ 2, avril, p. 60-70.

OPENA, C.L. (1983), Export Behavior and Incentives of Agribusiness Firms in the Philippines, Dissertation abstracts international volume 44/01-A, page 221, 181 pages.

PhILlipPe, J., (1990), dans Léo, P.Y., M.C. Monnayer-Longé et J. Phillipe, La PME: Stratégies internationales, Paris, Economica.

Ragman, M. et A. Verbeke (1989), « The impact of free trade on small business in Canada », $J S B E$, vol. 2, $\mathrm{n}^{\circ}$ 3, septembre, p. 51-56.

SCHWANEN, D. (1992), «Were the optimists wrong on free trade ? A canadian perspective », CD Howe Institute, $\mathrm{n}^{\circ} 87$, octobre, p. 11-24. 